\title{
Assistência de enfermagem ao recém-nascido submetido à diálise peritoneal na Unidade de Terapia Intensiva Neonatal
}

\author{
Nursing care for newborns unit undergoing peritoneal dialysis in the Neonatal Intensive \\ Care
}

\section{Cuidado de enfermería para recién nacidos sometidos a diálisis peritoneal en la Unidad de Cuidados Intensivos Neonatales}

Adriana Modesto Caxias $^{1 *}$, Rosangela de Jesus Nunes ${ }^{2}$, Danielle Serrão de Oliveira1 ${ }^{1}$, Marina Cristina da Silva Freitas ${ }^{3}$, Hallessa de Fátima da Silva Pimentel ${ }^{3}$, Thayná Gabriele Pinto Oliveira ${ }^{1}$, Alessandra Maria de Melo Cardoso ${ }^{1}$, Lozilene Amaral de Azevedo ${ }^{1}$, Kátia Silene Oliveira e Silva ${ }^{4}$, Daniel Tapajós de Freitas ${ }^{1}$.

\section{RESUMO}

Objetivo: Descrever temas relacionados à assistência de enfermagem ao recém-nascido internado na UTI Neonatal submetido à diálise peritoneal. Revisão bibliográfica: A diálise peritoneal (DP) é o método de primeira escolha para tratar a Lesão Renal Aguda em recém-nascidos que encontram-se na UTI Neonatal. E o enfermeiro, considerando legislação acerca de seu exercício, trabalha diretamente na promoção, proteção, recuperação e reabilitação da saúde de pacientes em situação de risco, estando estes profissionais diretamente envolvidos no cuidado ao neonato da UTI que encontra-se nesta condição. Assim, cabe ao profissional de enfermagem cuidados acerca da realização da DP, como a montagem e/ou troca dos sistemas (ou buretas), a troca das soluções dialíticas, a monitoração do balanço hídrico deste neonato, avaliação constante do aspecto dos curativos e do óstio do cateter, lavagem do cateter, controle contínuo dos sinais vitais e apoio psicológico à família. Considerações finais: Este estudo apresentou estratégias de cuidados de enfermagem com o RN em DP, como a monitorização de sinais vitais, cuidados com o cateter de tenckhoff e troca das soluções da DP, prevenção de infecção, monitoramento do equilíbrio hidroeletrolítico, entre outros, como forma de direcionar a atenção do enfermeiro para aspectos essenciais a serem avaliados em tais casos específicos.

Palavras-chave: Assistência de enfermagem, Recém-nascido, Diálise peritoneal.

\section{ABSTRACT}

Objective: Describe in the literature topics related to nursing care for newborns hospitalized in the Neonatal ICU undergoing peritoneal dialysis. Bibliographic review: Peritoneal dialysis (PD) is the method of choice to treat Acute Kidney Injury in newborns who are in the Neonatal ICU. And the nurse, considering legislation on their exercise, works directly in the promotion, protection, recovery and rehabilitation of the health of patients at risk, and these professionals are directly involved in the care of the newborn in the ICU who is in this condition. Thus, it is up to the nursing professional to care about the performance of PD, such as the assembly and/or exchange of systems (or burps), the exchange of dialysis solutions, the monitoring of the water balance of this newborn, constant evaluation of the aspect of the dressings and the catheter ostium, catheter washing, continuous control of vital signs and psychological support to the family. Final considerations: This study presented nursing care strategies with newborns in PD, such as monitoring vital signs, care with the tenckhoff catheter and exchange of PD solutions, infection prevention, monitoring of hydroelectrolytic balance, among others, as a way to direct nurses' attention to essential aspects to be evaluated in such specific cases.

Keywords: Nursing care, Newborn, Peritoneal dialysis.

\section{RESUMEN}

Objetivo: Describir en la literatura temas relacionados con la atención de enfermería para recién nacidos hospitalizados en la UCI Neonatal sometidos a diálisis peritoneal. Revisión bibliográfica: La diálisis peritoneal (PD) es el método de elección para tratar la lesión renal aguda en recién nacidos que se encuentran en la UCl

\footnotetext{
${ }^{1}$ Universidade da Amazônia (UNAMA), Belém - PA. *E-mail: adricoxufpa@yahoo.com.br

${ }^{2}$ Faculdade da Amazônia (FAMAZ), Belém - PA.

${ }^{3}$ Faculdade Pan Amazônica (FAPAN), Belém - PA.

${ }^{4}$ Universidade Federal do Pará (UFPA), Belém - PA.
} 
neonatal. Y la enfermera, considerando la legislación sobre su ejercicio, trabaja directamente en la promoción, protección, recuperación y rehabilitación de la salud de los pacientes en riesgo, y estos profesionales están directamente involucrados en el cuidado del recién nacido en la UCl que se encuentra en esta condición. Así, corresponde al profesional de enfermería preocuparse por el rendimiento de la DP, como el montaje y/o intercambio de sistemas (o burps), el intercambio de soluciones de diálisis, el seguimiento del equilibrio hídrico de este recién nacido, la evaluación constante del aspecto de los apósitos y el ostium del catéter, el lavado con catéter, el control continuo de signos vitales y el apoyo psicológico a la familia. Consideraciones finales: Este estudio presentó estrategias de cuidado de enfermería con recién nacidos en PD, tales como el monitoreo de signos vitales, la atención con el catéter tenckhoff y el intercambio de soluciones de DP, prevención de infecciones, monitoreo del equilibrio hidroelectrolítico, entre otros, como una manera de dirigir la atención de las enfermeras a aspectos esenciales a evaluar en estos casos específicos.

Palabras clave: Cuidado de enfermería, Recién nacido, Diálisis peritoneal.

\section{INTRODUÇÃO}

A diálise peritoneal (DP) é um dos principais procedimentos terapêuticos indicados para pacientes com Insuficiência Renal Aguda (IRA), ou Lesão Renal Aguda (LRA), como é chamada atualmente, por ser considerado um termo mais amplo, que inclui desde as mínimas alterações renais possíveis, até quadros mais graves acerca da patologia (CARVALHO KX, et al., 2015).

Considerando a importância de tais órgãos acometidos, os rins são componentes fundamentais do sistema urinário, consistindo em dois órgãos que possuem funções de grande importância para a regulação e manutenção do metabolismo humano, assim como excreção de escórias metabólicas e substâncias estranhas do organismo (TORTORA GJ e DERRICKSON B, 2016). Quando ocorre algum tipo de lesão em um ou ambos os órgãos, a principal consequência é o rápido comprometimento de sua função, acarretando no desenvolvimento da LRA (BRUNNER LS e SUDDARTH DS, 2015). Com a ocorrência da LRA, há um acúmulo de urina e eletrólitos, resultando em alto nível de concentração dessas substâncias no organismo (CERQUEIRA DP, et al., 2014).

Para Ferreira MJ, et al. (2013), dentre as terapias renais substitutivas existentes para o tratamento da LRA, há a DP, que consiste na inserção do líquido dialisador estéril na cavidade peritoneal com o objetivo de promover a troca de substâncias tóxicas por meio de difusão e osmose. Através desta terapia, o sangue que circula nos vasos sanguíneos do peritôneo entre em contato com o líquido dialisador inserido na cavidade abdominal por meio de um cateter (SANTOS J, 2013).

A DP é amplamente utilizada em recém-nascidos que desenvolvem LRA durante sua hospitalização em Unidade de Terapia Intensiva (UTI) Neonatal. E uma das hipóteses para o desenvolvimento da lesão em recém-nascidos - em especial os prematuros - seria o número reduzido de néfrons, o que implica na menor taxa de filtração glomerular (LOPES CB, et al., 2014).

Segundo a Sociedade Portuguesa de Pediatria (2016), cerca de 3 a $8 \%$ de recém-nascidos no mundo todo desenvolvem a lesão, dos quais estão em sua maioria os pré-termos, recém-nascidos com asfixia grave e os submetidos a cirurgia cardíaca. Ganha notoriedade quando relacionada à mortalidade neonatal devido seu alto índice, que pode ultrapassar a taxa de $25 \%$.

A UTI Neonatal onde estes recém-nascidos encontram-se é uma unidade de cuidados intensivos para neonatos com até 28 dias de vida que necessitam de hospitalização, com monitorização contínua e cuidados específicos, dispondo de aparato tecnológico complexo e recursos humanos especializados (COELHO AS, 2018).

A escassez acerca de assuntos relacionados à realização da DP na UTI Neonatal e o papel assistencial do enfermeiro ao longo do procedimento dialítico motivou a escolha do assunto, visto que este trabalho pretende ser colaborativo no aspecto da eficiência técnico-científica da assistência prestada ao recém-nascido nesta condição.

O presente estudo teve como objetivo descrever temas relacionados à assistência de enfermagem ao recém-nascido internado na UTI Neonatal submetido à DP, visto que cabe aos profissionais de saúde o 
cuidado ao longo do tratamento renal, e a assistência de enfermagem deve ser desenvolvida com vistas ao bom resultado do procedimento realizado, visando sempre contribuir para a melhoria na qualidade da assistência prestada a esse público.

\section{REVISÃO BILIOGRÁFICA}

\section{Lesão renal aguda (LRA) em recém-nascidos}

A Insuficiência Renal é uma síndrome em que ocorre o declínio da função renal, ocasionando efeitos nocivos ao organismo acometido, como: diminuição na taxa de filtração glomerular (TFG), falha para excretar os produtos residuais nitrogenados e dificuldade para manter a homeostasia hidroeletrolítica. Pode ser classificada em Crônica (desenvolve-se a longo prazo, como resultado de nefropatias anteriores que culminam com a degradação da função renal de forma gradativa. É clinicamente considerada irreversível). E em Aguda, que, ao contrário, pode ser reversível, podendo ocorrer em um curto período de tempo, como de horas a dias, tendo como principais causas o uso de medicamentos, infecções bacterianas, deposição de imunocomplexos e quadros hipovolêmicos (FERREIRA MJAS, et al., 2013).

A Insuficiência Renal Aguda passou a ser denominada de Lesão Renal Aguda (LRA), pois segundo a Sociedade Portuguesa de Pediatria (2016), o termo é mais abrangente em relação às diferentes causas que levam ao desenvolvimento desta patologia. Apresenta grande importância quando relacionado seu desenvolvimento em neonatos, visto que a taxa de mortalidade em recém-nascidos com LRA reside em torno de 25 a 50\%, sendo, assim, considerada alta, e afetando principalmente os que manifestam quadros de oligúria prolongada, falência multiorgânica ou necessidade de terapia substitutiva da função renal.

De acordo com Cloherty JP, et al. (2015), a LRA pode ser dividida em três formas, de acordo com a sua etiologia: Pré-renal (cardiopatia congênita, asfixia perinatal, enterocolite necrosante, choque, hemorragia, hipóxia, cirurgia cardíaca), Intríseca, por necrose tubular aguda (drogas nefrotóxicas, hipoperfusão, asfixia), por causas vasculares (trombose venosa ou arterial, necrose cortical), por pielonefrite e hemoglobinúria, e Pós-renal, com acometimento uretral (ureterocele, obstrução ureteropélvica, anomalia de junção ureteropélvica, refluxo vesicouretral primário), bexiga neurogênica, lesões compressivas extrínsecas (teratoma sacrococcígeo, hematocolpo), obstruções intrínsecas (benzoar fúngico), cálculo renal.

As causas de LRA em recém-nascidos são de difícil determinação, por apresentar variadas causas e com diferentes mecanismos de desenvolvimento da lesão renal. E dentre essas causas, está a reduzida taxa de filtração glomerular em recém-nascidos prematuros, devido ao menor número de néfrons que estes apresentam, visto que a nefrogênese se inicia a partir da $8^{\text {a }}$ semana de gestação e continua até a 34ª semana. Assim, quanto mais prematuro for o recém-nascido, menor será a quantidade de néfrons formados, sendo insuficientes para manter o equilíbrio homeostático do organismo (LOPES CB, et al., 2014).

De acordo com Carvalho KX, et al. (2015), a LRA em recém-nascidos pode iniciar ainda na vida intrauterina, e pode estar relacionada tanto a doenças congênitas como doenças genéticas, nesta situação. No período neonatal, mudanças volêmicas podem deixar o recém-nascido mais sensível ao desenvolvimento da patologia, pois é após o nascimento que os rins alcançam a maturação necessária para seu adequado funcionamento, apresentando cerca de dois milhões de néfrons em sua estrutura. Já no período pós-natal, as causas da LRA relacionam-se com frequência a insultos tóxicos e lesão hipóxica-isquêmica, sendo essa geralmente associada ao uso de antibióticos aminoglicosídeos e anti-inflamatórios não esteroides.

A principal característica presente em um neonato com LRA é a oligúria, em associação com alterações bioquímicas, como a presença de altas concentrações de produtos nitrogenados no sangue, acidose metabólica e distúrbios eletrolíticos (diferenças no metabolismo do potássio, hipernatremia, hipomagnessemia, hipofosfatemia, além de hematúria, proteinúria, uréia e creatina séricas aumentadas) (CARVALHO KX, et al., 2015). A oligúria em um recém-nascido é caracterizada por um débito urinário menor que 0,5 a $1 \mathrm{ml} / \mathrm{Kg} /$ hora no decorrer de 24 horas, ou mesmo com a ausência de diurese no mesmo período de tempo e, em especial, após 48 horas. É de fundamental importância que seja feita a monitorização do volume urinário através de sondagem vesical de demora para que o diagnóstico de IRA, junto à avaliação bioquímica do neonato, seja realizado corretamente (CLOHERTY JP, et al. 2015). 
E como forma de tratamento dessa patologia, o principal meio consiste na diálise, que busca remover os produtos residuais urêmicos acumulados no organismo acometido pelas lesões renais. $E$ dentre os tratamentos dialíticos existentes, os principais são a Hemodiálise e a Diálise Peritoneal, sendo esse último muito utilizado na UTI Neonatal (FERREIRA MJAS, et al., 2013).

\section{Diálise peritoneal (DP) na UTI neonatal}

Os cuidados realizados na UTI neonatal refletem a assistência multiprofissional de toda a equipe envolvida, sendo esta composta por profissionais enfermeiros, medicos, técnicos de enfermagem, fisioterapêutas, nutricionistas, psicólogos, entre outros. É o setor onde são prestados desde os cuidados mais simples até procedimentos de maior complexidade, onde os profissionais envolvidos precisam estar preparados tecnicamente, assim como atualizados em relação às informações mais atuais acerca de novas habilidades que possam ser desempenhadas no local, focando na segurança e bem estar do RN. E nesse cenário, um dos procedimentos realizados que mais demanda cuidado à equipe multiprofissional da UTI neo é a DP (NETO JDA, et al., 2016).

A DP foi utilizada pela primeira vez na década de 20, como forma de tratar a LRA. Contudo, foi apenas em 1946 que foi registrada a primeira vida salva após a realização do tratamento em paciente com essa patologia. A princípio, as soluções utilizadas na DP começaram a provocar hipercloremia e hipervolemia, sendo necessários alguns ajustes para que os resultados fossem satisfatórios. Da mesma forma, os cateteres utilizados também foram aprimorados. E, a partir de então, a utilização da DP aumentou gradativamente (CULLIS B, et al., 2014).

Pode ser classificada de acordo com o tipo de realização do tratamento, sendo esses: Diálise Peritoneal Ambulatorial Contínua (CAPD) - pode ser realizada no domicílio pelo próprio paciente ou por uma pessoa da família devidamente treinada -, Diálise Peritoneal Intermitente (IPD) - é indicado para pessoas com náuseas, vômitos, fadiga, estado mental alterado, sobrecarga hídrica, acidose e hipercalemia - e Diálise Peritoneal Cíclica Contínua (CCPD) - há a combinação da IPD durante a noite com um tempo de retenção prolongado durante o dia (CULLIS B, et al., 2014).

A DP é constituída de três componentes importantes (fluxo sanguíneo, membrana peritoneal e solução de diálise), sendo processada em três fases, que são: infusão da solução, permanência e drenagem da solução, onde os períodos de tempo para a realização da troca são fixos e de acordo com a modalidade a ser utilizada (WASEN PE, et al., 2015).

Trata-se de um método dialítico de primeira escolha para o tratamento de LRA na UTI neonatal, onde o tratamento consiste no manejo de solução de glicose e eletrólitos na cavidade peritoneal por meio do cateter de tenckhoff, onde o peritônio - membrana semipermeável da cavidade peritoneal - irá funcionar como um filtro, possibilitando a troca de solutos entre os meios por osmose e difusão. Nesse processo, ocorre a passagem de água, eletrólitos e toxinas pelos capilares da membrana peritoneal, de forma passiva, para 0 fluído de diálise por meio da drenagem. E sua finalidade é a remoção de metabólitos endógenos, toxinas exógenas e excesso de fluído do organismo, tal como ocorre na função renal (CARVALHO KX, et al., 2015).

O cateter de Tenckhoff inserido possui dois cuffs, sendo que um se prende ao tecido subcutâneo e o outro à parede reto abdominal, onde ambos apresentam função bacteriostática. Posteriormente, o dispositivo é fixado internamente com a produção e deposição de fibrina no local (SANTANA NC, et al., 2016).

Em recém-nascidos - e até mesmo em crianças -, o sistema de DP utilizado é o manual, cujo circuito deve ser fechado no sentido de reduzir ao máximo o risco de infecção. Além disso, o controle do volume administrado é de suma importância para minimizar o risco de distensão abdominal, a qual, caso ocorra, pode ter como consequências a restrição respiratória ou vazamento do líquido da drenagem. E ainda segundo este autor, as soluções de DP para tratamento de LRA são compostas de dextrose em concentração suficiente para atingir o alvo de ultrafiltração (CULLIS B, et al., 2014).

E por se tratar de um procedimento invasivo, o risco de complicações na realização da DP está sempre presente, principalmente se não for realizada de maneira correta. Dentre as complicações que podem ocorrer, 
estão as mecânicas (dor abdominal e torácica, sangramento, extravasamento, fluxo inadequado, perfuração de vísceras, obstrução do cateter), infecciosas (peritonite) e clínicas (hipervolemia, hipovolemia, hiperglicemia, perda proteica, entre outros). Contudo, os benefícios que a DP traz ao recém-nascido com LRA superam os risco de complicações, devido a efetividade de seu tratamento (CARVALHO KX, et al., 2015).

Assim, como parte do processo de enfermagem, a assistência do enfermeiro na DP é de suma importância, pois seus cuidados e intervenções podem minimizar - ou mesmo anular - riscos que possam vir a ocorrer durante o procedimento (SILVA RAR, et al., 2016).

\section{Assistência de enfermagem}

Considerando a Lei ํㅜ 7.498, de 25 de junho de 1986, a qual trata acerca da regulamentação do Exercício da Enfermagem, este profissional possui, dentre suas várias atribuições:

"Art. 11. [...] I- privativamente: [...] c) planejamento, organização, coordenação, execução e avaliação dos serviços de assistência de enfermagem", [...] I) cuidados diretos de enfermagem a pacientes graves com risco de vida" e " $m$ ) cuidados de enfermagem de maior complexidade técnica e que exijam conhecimentos de base científica e capacidade de tomar decisões imediatas" (BRASIL, 2009).

Dessa forma, pode-se inferir que o profissional de enfermagem trabalha diretamente na promoção, proteção, recuperação e reabilitação da saúde de pacientes em situações de risco, de forma individual e sempre considerando a peculiaridade de cada situação (BRASIL, 2009). Assim, a atuação da enfermagem na TRS de DP exige desses profissionais um amplo conhecimento acerca do desempenho de habilidades técnicas e científicas, pois estão diretamente envolvidos no cuidado ao neonato da UTI que encontra-se nesta condição, prestando assistência de forma individualizada, integral e humanizada (CARVALHO KX, et al., 2015).

E, considerando a legislação vigente, cabe ao profissional enfermeiro - em relação a TRS - a montagem e/ou troca do sistema de bolsas (ou buretas) da DP do recém-nascido que encontra-se na UTI neonatal, devido ao número de conexões para que o fluxo ocorra de forma correta ser um sistema complexo, necessitando de um profissional habilitado técnica e cientificamente para tal. E, segundo Santana NC, et al. (2016), deve-se dar preferência ao uso de técnicas com o menor número de conexões possíveis, à um sistema fechado e à realização do "flush before fill", que consiste na escovação do sistema antes do início da infusão com o intuito de reduzir o risco de contaminação.

Os cuidados, de modo geral, baseiam-se no antes e após a realização da DP, com a manutenção de todos os tubos clampeados após serem cheios com a solução dialisadora, sendo abertos somente no momento em que for iniciar a terapia. (TAMEZ RN, 2017). A troca das soluções dialíticas também compete ao enfermeiro, e segundo Cullis B, et al. (2014), deve-se ter atenção para a mensuração das concentrações séricas de eletrólitos, as quais devem ser realizadas a cada 12 horas nas primeiras 24 horas e, assim que os parâmetros estiverem estáveis, devem ser realizadas diariamente. Da mesma forma, o balanço hídrico deve ser realizado durante o tratamento pelo enfermeiro, considerando o volume recebido e suas perdas, incluindo as insensíveis. E como o balanço hídrico nesses pacientes deve ser feito de modo rigoroso, é indicado o uso de buretas que, além de permitir a medida precisa de ambos os fluxos, diminui o número de conexões, assim como o risco de contaminação por contato.

Para Silva RAR, et al. (2016), a monitoração do balanço hídrico pelo enfermeiro também consiste em observar o volume de líquidos excessivos nos espaços tissulares. Isso pode ocorrer em neonatos em DP devido a descompensação dos mecanismos regulatórios, entre as pressões coloidosmótica e hidrostática, que compõem o leito peritoneal. E como intervenções deste profissional, pode-se citar o controle hídrico, controle hidroeletrolítico e monitoração hídrica, a partir da restrição de líquidos, avaliação da presença de edema e continuidade da realização de balanço hídrico.

Em relação ao cateter de tenckhoff, embora inserido por um médico cirurgião após o recém-nascido ser submetido a uma pequena cirurgia em um centro cirúrgico ou à beira do leito - em casos realmente 
necessários -, utilizando a técnica de Seldinger no ambiente mais estéril possível, cabe ao enfermeiro prestar a devida assistência ao neonato em uso desse dispositivo, visto que após a sua fixação, esse profissional terá os devidos cuidados com o local da inserção, realizando o curativo oclusivo após o procedimento cirúrgico, utilizando solução salina ou clorexidina aquosa, deixando-o seco e estéril posteriormente, para sua manutenção no período de epitalização (SANTANA NC, et al., 2016).

O enfermeiro deve avaliar constantemente o aspecto dos curativos e do óstio do cateter, assim como atentar para qualquer anormalidade deste orifício, com vistas a prevenir futuras complicações que possam ocorrer, como, por exemplo, infecções (peritonite) ou complicações mecânicas que possam prejudicar a continuidade do tratamento. Por isso, é necessário que haja um ambiente apropriado e higienizado para a realização dos curativos, a organização e antissepsia de todo o material que será utilizado, higienização correta das mãos, com abertura e fechamento de torneira utilizando-se os cotovelos, uso de produtos indicados à limpeza do cateter, uso de EPI's para o procedimento (máscara, luvas estéreis e capote) uma correta e contínua higiene do orifício de entrada, fixação e cuidados com o manuseio do dispositivo para não haver riscos de contaminação (WASEN PE, et al., 2015). Para Santana NC, et al. (2016), a higienização das mãos deve ser rigorosa antes da manipulação do cateter.

A lavagem do cateter de Tenchoff através da realização de flush é um procedimento que poderá ser realizado somente pelo enfermeiro, quando esse for prescrito pelo médico assistente, sendo que o profissional de enfermagem deve estar apto para tal ato e sinta-se seguro quanto a sua realização (BRASIL, 2009).

Por se tratar de um procedimento invasivo, a DP pode apresentar complicações, que podem ser detectadas, ou mesmo evitadas pelo enfermeiro através de uma assistência efetiva ao neonato. $E$ dentre as complicações que podem surgir, pode-se considerar a mais frequente em pacientes em TRS de DP a peritonite, que consiste na inflamação do peritônio (SILVA RAR, et al., 2016). É considerada a principal causa de interrupção do tratamento dialitico, visto que quando a inflamação torna-se prolongada ou severa, pode causar danos na membrana peritoneal (BALBI AL, et al., 2017)

E como forma de minimizar os riscos de infecções, esse profissional deve avaliar o estado imunológico desse neonato, com a meta de manter esse estado adequado através da identificação dos riscos iminentes, ou por meio da higienização correta do cateter e região próxima a ele, além de medidas técnicas e comportamentais, como uso de técnicas assépticas sempre que for manusear o local da inserção (SILVA RAR, et al., 2016).

O enfermeiro deve realizar o controle contínuo dos sinais vitais visando também evitar outras complicações que podem surgir ao longo do tratamento, como a hipotensão, que pode estar associada à redução excessiva do volume sanguíneo, perda da vasoconstrição e fatores cardíacos, ou mesmo a hipertensão, que pode ser causada pela sobrecarga hídrica, resposta da renina à ultrafiltração, ou mesmo pelo excesso de sódio, entre outros (CLOHERTY JP, et al. 2015).

E por se tratar de pacientes em uma situação complexa, a equipe de enfermagem deve estar preparada, com conhecimentos teórico-práticos específicos, com o intuito de atender esses neonatos no ambiente hospitalar com segurança, prevenindo iatrogenias que possam ocorrer e/ou consequências que possam colocar em risco a continuidade do tratamento dialítico. Nesse contexto, o enfermeiro capacitado deve estar à frente de uma educação continuada junto à equipe de enfermagem, de forma permanente, com o objetivo de atualizar e capacitar a equipe frente às dificuldades sociais e evoluções técnicas e científicas. Além disso, a própria experiência do profissional enfermeiro envolvido no desenvolvimento da DP pode somar de forma positiva na aquisição de novas competências para o alcance do sucesso do tratamento (CARVALHO KX, et al., 2015).

O enfermeiro também está diretamente envolvido no apoio psicológico dado à família do recém-nascido, visto que esse profissional deve facilitar o entendimento da situação e possibilitar, dentro do possível, o bemestar emocional, físico e espiritual dos membros e da unidade familiar (WASEN PE, et al., 2015).

Brunner LS e Suddarth DS (2015) apresentam em seu trabalho a assistência de enfermagem ao paciente com LRA (Quadro 1): 
Quadro 1 - Assistência de Enfermagem ao Recém-Nascido com LRA em tratamento de DP.

\begin{tabular}{|l|l|}
\hline \multicolumn{2}{|l|}{$\quad$ Manejo de Enfermagem } \\
\hline 1 & Monitorar as complicações; ajudar no tratamento de emergência dos distúrbios hidreletrolíticos. \\
\hline 2 & Avaliar a evolução e a resposta do neonato ao tratamento. \\
\hline 3 & $\begin{array}{l}\text { Manter a família informada a respeito da condição do recém-nascido; isso ajuda a família a } \\
\text { compreender os tratamentos e fornece apoio psicológico. }\end{array}$ \\
\hline 4 & Continuar fornecendo o cuidado de enfermagem indicado para o distúrbio primário. \\
\hline & \multicolumn{2}{|l|}{$\begin{array}{l}\text { Monitorar os níveis séricos dos eletrólitos, bem como os indicadores físicos dessas complicações, } \\
\text { durante todas as fases do distúrbio. }\end{array}$} \\
\hline 2 & $\begin{array}{l}\text { Triagem dos líquidos parenterais, toda a ingestão oral (ou por sonda) e todos os medicamentos à } \\
\text { procura de fontes ocultas de potássio. }\end{array}$ \\
\hline 3 & Monitorar a função cardíaca e o estado musculoesquelético à procura de sinais de hiperpotassemia. \\
\hline 4 & $\begin{array}{l}\text { Atentar para aporte de líquidos (os medicamentos IV devem ser administrados com o menor volume } \\
\text { possível), débito urinário, edema aparente, distensão das veias jugulares, alterações das bulhas } \\
\text { cardíacas e sons respiratórios, e dificuldade crescente na respiração }\end{array}$ \\
\hline 5 & Manter registros precisos do equilíbrio hídrico e peso diário \\
\hline 6 & Relatar, imediatamente, os indicadores de deterioração do estado hidreletrolítico. \\
\hline 7 & $\begin{array}{l}\text { Preparar o recém-nascido para o tratamento de emergência da hiperpotassemia e para diálise } \\
\text { peritoneal, quando indicada, para corrigir os desequilíbrios hidreletrolíticos. }\end{array}$ \\
\hline
\end{tabular}

A hiperpotassemia constitui o distúrbio potencialmente fatal mais imediato observado na LRA. Os líquidos parenterais, toda a ingestão oral e todos os medicamentos são cuidadosamente rastreados para garantir que fontes de potássio não sejam inadvertidamente administradas ou consumidas.

Redução da Taxa Metabólica

- Evitar ou tratar imediatamente a febre e a infecção.

Promoção da Função Pulmonar

- Ajudar o neonato durante o seu manuseio, realizar mudança de decúbito,

Prevenção da Infecção

- Praticar assepsia quando estiver trabalhando com linhas invasivas e cateteres.

Cuidados da Pele

- Realizar meticuloso cuidado da pele.

- Realizar a higiene corporal do neonato, efetuar mudanças frequentes de decúbito, manter a pele limpa e bem umidificada.

Cuidados da Pele

- Proporcionar apoio à família do recém-nascido durante a diálise peritoneal (DP); não omitir as necessidades psicológicas e as preocupações.

- Explicar a finalidade do tratamento à sua família. Repetir as explicações e esclarecer as perguntas, quando necessário.

- Incentivar a família a tocar o neonato e a conversar com ele durante a DP.

- Avaliar continuamente o recém-nascido à procura de complicações da IRA e suas causas precipitantes.

Fonte: Caxias AM, et al., 2020, baseado em Brunner LS e Suddarth DS, 2015.

O profissional de enfermagem necessita ter, além da fundamentação científica e da competência técnica, conhecimento dos aspectos que levam em consideração os sentimentos da família e as necessidades do neonato durante o tratamento dialítico. Assim, o papel do enfermeiro é destacado como de grande colaboração, pois busca o bem-estar do recém-nascido, assistindo-o holisticamente, e prestando a devida assistência tanto ao neonato como a sua família, e trabalhando sempre em conjunto com o restante da equipe (CARVALHO KX, et al, 2015). 


\section{CONSIDERAÇÕES FINAIS}

As ações de enfermagem para que uma eficiente assistência seja prestada ao recém-nascido com LRA em tratamento de DP tem como necessidade considerar seu tratamento como um todo e os riscos a que esse neonato possa estar exposto. Nesse sentido, as intervenções de enfermagem devem ser implementadas visando contribuir para o delineamento de diferentes ações clínicas neste contexto. Dessa forma, este estudo apresentou estratégias de cuidados de enfermagem com o RN em DP, como a monitorização de sinais vitais, cuidados com o cateter de tenckhoff e troca das soluções da DP, prevenção de infecção, monitoramento do equilíbrio hidroeletrolítico, apoio psicológico à família, entre outros,, sendo de suma importância para alicerçar a prática clínica do enfermeiro, como forma de direcionar a atenção desse profissional para aspectos essenciais a serem avaliados em tais casos específicos, que, além de propor melhorias na assistência prestada de maneira a contribuir para uma resposta positiva ao tratamento dialítico, também abordou planos de cuidados relacionados à patologia em si.

\section{REFERÊNCIAS}

1. BALBI AL, et al. Protocolos Clinicos e Padronização de Condutas em Diálise. Hospital das Clinicas da Faculdade de Medicina de Botucatu - HCFMB. Universidade Estadual Paulista "Júlio de Mesquita Filho". Botucatu., 2017; 120p.

2. BRASIL. 2009. Lei no 7.498 , de 25 de junho de 1986. Dispõe sobre a regulamentação do exercício da enfermagem, e dá outras providências. Presidência da República, Brasília, DF, 21 set. 2009. Disponivel em: http://www.planalto.gov.br/ccivil_03/leis/L7498.htm. Acesso em: 15 abr. 2019.

3. BRUNNER LS, SUDDARTH DS. Manual de Enfermagem médico-cirúrgica / Revisão Técnica: Sônia Regina de Souza; Tradução: Patrícia Lydie Voueux. - 13 ed. - Rio de Janeiro: Guanabara Koogan, 2015; 1.152p.

4. CARVALHO KX, et al. O cuidado ao recém-nascido submetido à diálise peritoneal: desafios para a equipe de enfermagem. Cogitare Enferm., 2015; 20(1): 139-145.

5. CERQUEIRA DP, et al. Fatores preditivos da Insuficiência renal e algoritmo de controle e tratamento. Rev. Latino-Am. Enfermagem., 2014; 22(2): 211-217.

6. CLOHERTY JP, et al. MANUAL DE NEONATOLOGIA. Guanabara Saúde Profissional, 2015; 7: 1.033p.

7. COELHO AS, et al. Equipe de enfermagem e a assistência humanizada na UTI neonatal. ReonFacema, $2018 ; 4: 1$.

8. CULLIS B, et al. Peritoneal dialysis for acute kidney injury. Peritoneal dialysis international, 2014; 34(5): $494-517$.

9. FERREIRA MJAS, et al. O cuidado à criança com insuficiência renal: uma revisão integrativa da literatura. Cadernos de Graduação - Ciências Biológicas e da Saúde Facipe, 2013; 1(1): 37-49.

10. LOPES CB, et al. Insuficiência Renal Aguda em uma Unidade de Terapia Intensiva no Norte do Brasil. Revista Paraense de Medicina, 2014; 28(1).

11. NETO JDA, et al. Profissionais de Saúde da Unidade de Terapia Intensiva: Percepção dos fatores restritivos da atuação multiprofissional. Revista Brasileira em Promoção da Saúde, 2016; 29(1): 43 - 50.

12. SANTANA NC, et al. Cuidados de Enfermagem com o Cateter de Tenckhoff frente a prevenção da peritonite em Diálise Peritoneal. In: 18ª SEMANA DE PESQUISA DA UNIVESIDADE TIRADENTES, 2016, Aracajú. Anais, 2016; 18072158.

13. SANTOS J. Repercussões de Diálise Peritoneal em crianças assistidas em UTI Pediátrica, um desafio para enfermeiros e família. Faculdade Meta - FAMETA. Pós-graduação em UTI Pediátrica e Neonatologia. Rio Branco, Acre, 2013.

14. SILVA RAR, et al. Diagnóstico, resultados e intervenções de enfermagem para pacientes em diálise peritoneal. Acta Paul Enferm., 2016; 29(5): 486-93.

15. SOCIEDADE PORTUGUESA DE PEDIATRIA. Lesão Renal Aguda no Recém-nascido. Secção de Neonatologia. Consenso Clínico, 2016; 1(1); 1-15.

16. TAMEZ RN. Enfermagem na UTI Neonatal: assistência ao recém-nascido de alto risco. 6. ed. - Rio de Janeiro: Guanabara Koogan, 2017; 394p.

17. TORTORA GJ, DERRICSON B. Princípios de Anatomia e Fisiologia. Tradução: Ana Cavalcanti C. Botelho ... [et al]. 14. ed. Rio de Janeiro: Guanabara Koogan, 2016; 1.598p.

18. WASEN PE, et al. Refletindo acerca dos cuidados de enfermagem de crianças em diálise peritoneal e suas famílias: uma revisão integrativa. Revisa Cuidado em Enfermagem - CESUCA. 2015; 1(1); 13-39. 